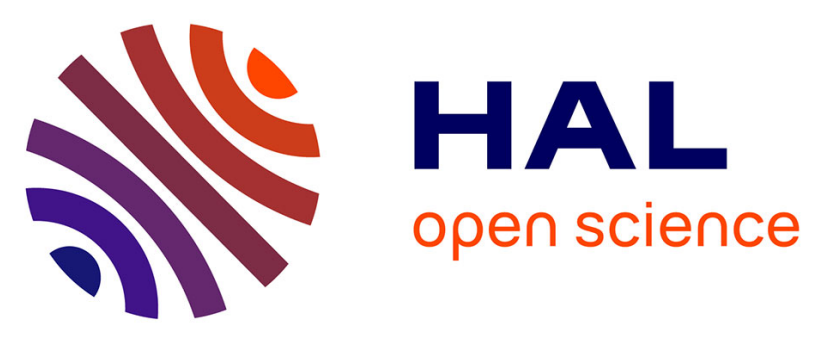

\title{
Nanoparticle Uptake in Plants: Gold Nanomaterial Localized in Roots of Arabidopsis thaliana by X-Ray Computed Nanotomography and Hyperspectral Imaging
} Astrid Avellan, Fabienne Schwab, Armand Masion, Perrine Chaurand, Daniel

Borschneck, Vladimir Vidal, Jérôme Rose, Catherine Santaella, Clément

\author{
Levard
}

\section{To cite this version:}

Astrid Avellan, Fabienne Schwab, Armand Masion, Perrine Chaurand, Daniel Borschneck, et al.. Nanoparticle Uptake in Plants: Gold Nanomaterial Localized in Roots of Arabidopsis thaliana by XRay Computed Nanotomography and Hyperspectral Imaging. Environmental Science and Technology, 2017, 51, pp.8692-8691. 10.1021/acs.est.7b01133 . hal-01566237

\section{HAL Id: hal-01566237 \\ https://hal.science/hal-01566237}

Submitted on 11 May 2018

HAL is a multi-disciplinary open access archive for the deposit and dissemination of scientific research documents, whether they are published or not. The documents may come from teaching and research institutions in France or abroad, or from public or private research centers.
L'archive ouverte pluridisciplinaire HAL, est destinée au dépôt et à la diffusion de documents scientifiques de niveau recherche, publiés ou non, émanant des établissements d'enseignement et de recherche français ou étrangers, des laboratoires publics ou privés. 


\title{
Nanoparticle Uptake in Plants: Gold Nanomaterial Localized in Roots of Arabidopsis thaliana by X-ray Computed Nanotomography and Hyperspectral Imaging
}

Astrid Avellan ${ }^{\dagger \ddagger \S} \quad$, Fabienne Schwab ${ }^{\dagger \ddagger}$, Armand Masion $^{\dagger \ddagger}$, Perrine Chaurand $^{\dagger \ddagger}$, Daniel Borschneck $^{\dagger \ddagger}$, Vladimir Vidal ${ }^{\dagger \ddagger}$, Jérôme Rose ${ }^{\dagger \ddagger}$, Catherine Santaella ${ }^{\ddagger \S}$, and Clément Levard ${ }^{* \ddagger}$

\author{
${ }^{+}$Aix Marseille Université, CNRS, IRD, College De France, CEREGE, Aix en Provence, France \\ ${ }^{\ddagger}$ iCEINT, International Center for the Environmental Implications of \\ Nanotechologies, CNRS-Duke University, Europôle de I'Arbois, 13545 Aix-en- \\ Provence, France \\ ${ }^{\S}$ Aix Marseille Université, CEA, CNRS, Laboratory of Microbial Ecology of the Rhizosphere \\ and Extreme Environments (LEMIRE), Biosciences and Biotechnology Institute of Aix- \\ Marseille (BIAM) ECCOREV, FR 3098, CEA/Cadarache, St-Paul-lez-Durance, France \\ Environ. Sci. Technol., 2017, 51 (15), pp 8682-8691
}

\section{Abstract}

Terrestrial plants can internalize and translocate nanoparticles (NPs). However, direct evidence for the processes driving the NP uptake and distribution in plants is scarce at the cellular level. Here, NP-root interactions were investigated after 10 days of exposure of Arabidopsis thaliana to $10 \mathrm{mg} \cdot \mathrm{L}^{-1}$ of negatively or positively charged gold NPs ( $\left.\sim 12 \mathrm{~nm}\right)$ in gels. Two complementary imaging tools were used: X-ray computed nanotomography (nanoCT) and enhanced dark-field microscopy combined with hyperspectral imaging (DF-HSI). The use of these emerging techniques improved our ability to detect and visualize NP in plant tissue: by spectral confirmation via DF-HSI, and in three dimensions via nano-CT. The resulting imaging provides direct evidence that detaching border-like cells (i.e., sheets of border cells detaching from the root) and associated mucilage can accumulate and trap NPs irrespective of particle charge. On the contrary, border cells on the root cap behaved in a charge-specific fashion: positively charged NPs induced a higher mucilage production and adsorbed to it, which prevented translocation into the root tissue. Negatively charged NPs did not adsorb to the mucilage and were able to translocate into the apoplast. These observations provide direct mechanistic insight into NP-plant interactions, and reveal the important function of border cells and mucilage in interactions of plants with charged NPs.

\section{Introduction}

Understanding the interactions of nanoparticles (NPs) with terrestrial plants is essential to predict their fate in terrestrial environments and their possible accumulation in the food chain.(1) Plant roots can internalize and accumulate NPs, presumably primarily via the apoplastic pathway along the cell walls and intercellular spaces into the vasculature. From the vasculature, NPs may further translocate into stems and leaves. $(2,3)$ Initial studies on metal- 
based NP interactions with roots suggest a charge-dependent NP uptake and translocation rates. Positively charged NPs have been found to associate to a greater extent with roots. Negatively charged NPs can be taken up more easily and translocate more efficiently into shoots.(4-9) Until now, phytotoxicity of NPs has gained more interest than the underlying mechanisms of NP uptake and the associated mobility in plants.(10)

A major challenge in studying NP-plant interactions is to obtain direct and unambiguous evidence of NP uptake.(11) Initial studies assessed NP-root association and translocation via metal analysis in roots and shoots, and visualization tools such as electron microscopy to confirm the NP uptake. Using a single visualization technique to localize NPs in plant tissue often results in conflicting data, especially at low, environmentally relevant concentrations. The few observable NPs can be almost indistinguishable from naturally occurring NPs or other background signals. $(12,13)$ Moreover, complex and destructive sample preparation protocols as labeling, staining, sputter-coating, and ultrathin cutting, can all introduce artifacts.(14) Less invasive sample preparation and the coupling of complementary techniques, e.g., elemental analysis coupled with NP identification and mapping techniques, can help to reduce such artifacts.(15)

One emerging technique with great potential to be included in such an interdisciplinary approach is the enhanced resolution dark-field microscopy(16) and hyperspectral imaging (DF-HSI). This 2D visualization tool requires minimal sample preparation, and can detect and map the NP-specific reflectance spectra of a material in complex environmental matrices(17) at the nanoscale, in relatively short time (minutes-hours), and in a narrow focus plane. The current spectral resolution of DF-HSI is $1.5 \mathrm{~nm}$, and the spatial resolution is about $90 \mathrm{~nm} .(16,17)$ Importantly, although 3D imaging could be done with the most recent setups, DF-HSI mainly enabled 2D visualization and could fail in identifying NPs biological barrier crossing. Objects smaller than the spatial resolution $(\sim 10 \mathrm{~nm})$ can also be detected if they possess a strong light scattering signal. Dark-field hyperspectral imaging has already been used to study NP-organism interactions, e.g., in vitro interactions of NPs with cell; $(18,19)$ or in vivo interactions of NPs with unicellular organisms such as protozoa,(20) bacteria,(2123) and green algae,(24, 25) or in entire organisms such as fishes(26) or worms.(27, 28) Despite its demonstrated usefulness to provide information on NP location in cells and small organisms, DF-HSI has not yet been used to detect NPs in terrestrial plants.

An other promising technique for NP imaging in plants providing valuable complementary information besides DF-HSI is X-ray computed tomography (CT). This mature 3-dimensional (3D) imaging technique is based on the $X$-ray attenuation by a sample. CT requires neither cutting nor labeling or staining of the samples, which greatly reduces the risk of artifacts from 
sample preparation. CT imaging techniques have resolutions of $\sim 1 \mu \mathrm{m}$ and $\sim 50 \mathrm{~nm}$ for microand nano-CT, respectively. Micro-CT has been successfully used to visualize microscopic plant features in 3D with a resolution of few $\mu \mathrm{m}$.(29) Nano-CT was first developed on synchrotron beamlines,(30)and has recently been adapted to benchtop systems.(31, 32) Nano-CT could provide valuable 3D information with a good resolution on the roots-NP interaction (adsorption vs internalization), and the scanning of large volumes can simplify the detection of low NP concentrations. However, unambiguous identification of NPs can become challenging in natural heterogeneous sample potentially composed of impurities with X-ray attenuation similar to that of metal-based NPs. A cross-validation identification with a NPspecific technique is then required.

In the present study, a novel methodology based on these two complementary 2D and 3D imaging techniques (DF-HSI and nano-CT) was proposed to perform the characterization of NP-plant interactions at the cellular level. We aimed to explore the capabilities and limitations of the combination of DF-HSI and nano-CT to obtain two and three-dimensional and crossvalidated information on distribution of small ( $12 \mathrm{~nm}$ in diameter) Au-NPs on and in plant roots. Arabidopsis thaliana served as a model plant and was exposed to negatively and positively charged gold NPs ( $\mp$ Au-NPs). This approach allowed an evaluation of hypotheses on NP uptake mechanisms at the cellular level.

\section{Experimental Section}

\subsection{Au-NPs}

Negatively ((-)Au-NPs) or positively ((+)Au-NPs) charged Au-NPs stabilized by a coating of citrate, or branched polyethylenimine, respectively, were purchased from nanoComposix Inc., Czech Republic. Diameters of (₹)Au-NPs provided by the manufacturer were $13.4 \pm 1.3 \mathrm{~nm}$, and $12.1 \pm 0.8 \mathrm{~nm}$, respectively. TEM images and distribution histograms are shown in Figure S1 of the Supporting Information (SI). Zeta potentials and hydrodynamic diameter of the NPS were measured by electrophoretic- and dynamic light scattering (Zetasizer nanozS, Malvern Inc., U.K.). The resulting hydrodynamic diameters and zeta potential for (-)Au-NPs and (+)AuNPs at $\mathrm{pH} 7 \pm 0.2$ in stock suspensions diluted in ultrapure water, were $18.6 \pm 7.1 \mathrm{~nm}$ and $-32.1 \mathrm{mV}$; and $47.6 \pm 11.3 \mathrm{~nm}$ and $+46.3 \mathrm{mV}$, respectively. The latter measurements confirmed those provided by the manufacturer.

\subsection{Plant Culture, Exposure to Au-NPs and Growth}

Arabidopsis thaliana (Columbia ecotype) seeds were grown in gel as described elsewhere.(33)Seeds were surface-sterilized for $5 \mathrm{~min}$ in Tween 20, rinsed twice with ethanol, and air-dried sterilely. Ten seeds were sown in square plates $\left(12 \times 12 \mathrm{~cm}^{2}\right)$ containing sterile 
one-half-strength Hoagland's solution solidified with Phytagel (Sigma-Aldrich, United States). Recipes of these solutions are shown in Table S1 in the SI).

Au-NPs were mixed with the nutrient/Phytagel solution prior to solidification to obtain a

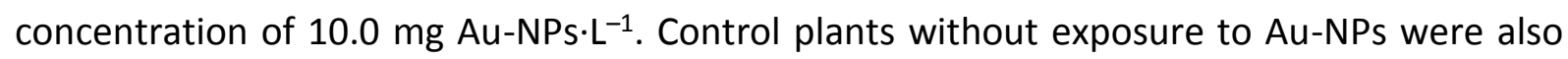
prepared. The plates were sealed with Micropore tape (3M, U.S.A.) to limit water evaporation and allow gas exchange, and incubated vertically for 10 days at a photon flux from the top of $150 \mathrm{\mu mol} \cdot \mathrm{m}^{-2} \mathrm{~s}^{-1}$ and under a light/dark cycle of $16: 8 \mathrm{~h}$ and $21: 19{ }^{\circ} \mathrm{C}$. The germination rate was determined on ten seeds 10 days postsowing, and the length of the roots measured using digital images and ImageJ software.(34)

\subsection{Preparation of Roots for Analysis}

Per treatment, five plants were analyzed in total: two plants by DF-HSI, one by nano-CT, and two by $\mu$-XRF. For DF-HSI, the roots were separated from the shoots using a sterile razor blade immediately prior to analysis, rinsed 3 times with a sterilized solution containing $10^{-3} \mathrm{~mol} \mathrm{~L}^{-}$ ${ }^{1} \mathrm{KCl}$, and were directly mounted between a glass slide and coverslip with a $200 \mu \mathrm{L}$ drop of the $\mathrm{KCl}$ solution.

For $\mu$-XRF and nano-CT root analysis, harvested roots were washed three times for 5 min with pure sodium phosphate buffer (PPB, 0.10M, pH 7.2) and fixed using $2.5 \%(\mathrm{v} / \mathrm{v})$ glutaraldehyde in PPB at room temperature for $12 \mathrm{~h}$. Fixed roots were then dehydrated by immersion in ethanol series of $25,50,70,90,90,100$, and 100 (\% v/v) for $20 \mathrm{~min}$ each. The ethanolsaturated roots were dried using a $\mathrm{CO}_{2}$ supercritical point dryer (EM CPD 3000, Leica Microsystems Inc., U.S.A.) to preserve the cellular structure of the root tissue, and to limit drying artifacts.(35) Dried roots were then slipped into a polyimide tube (Kapton, ColeParmer, U.S.A.) mounted on a pin-type sample holder. For $\mu$-XRF measurements, the supercritical point dried roots were fixed with polyimide tape on a clean silicon wafer.

\subsection{Elemental Microanalysis of Roots by $\mu$-XRF}

We performed micro-X-ray fluorescence ( $\mu$-XRF) on two different roots to identify the root regions containing the highest $\mathrm{Au}$ content. Semiquantitative analysis of $\mathrm{Ca}$ and $\mathrm{Au}$ in roots was performed using a custom-built laboratory-scale $\mu$-XRF microscope named HERMES (High Xray Energy Resolution Microscope for Environmental Sciences). High sensitivity was achieved through a high flux incident $\mathrm{X}$-ray beam (Mo rotating anode, $50 \mathrm{kV}, 24 \mathrm{~mA}, 1-2 \times 10^{11}$ photons $\mathrm{mm}^{-2} \mathrm{~s}^{-1}$, spot diameter of $400 \mu \mathrm{m}$, high-flux optics from XENOCS, Chantilly, U.S.A.), and a 4 elements X-ray detector (Vortex-ME4, Hitachi, Japan), allowing for the detection of relatively low local element concentrations ( $<50$ ppm). 
Two positions on the root apex were selected and analyzed: (i) the root tip, i.e., the apical zone of cell division including the root cap; and (ii) $\sim 1 \mathrm{~cm}$ above the root tip, the elongation zone including the young vasculature (Figure S2). $\mu$-XRF spectra were collected using a counting time of $1800 \mathrm{~s}$. In our case, X-ray fluorescence was not able to discriminate between sorption on the root surface vs root internalization because (i) at this energy, the incoming $X-$ ray beam fully penetrated the samples, and (ii) the X-ray spot diameter $(400 \mu \mathrm{m})$ was bigger than the root diameter (about $100 \mu \mathrm{m}$ ).

Semiquantitative comparison was performed between the apex and the elongation zone by

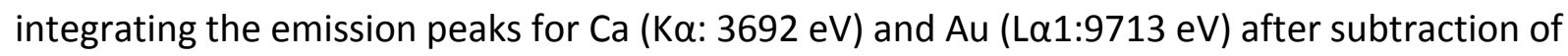
the sample holder (silicon wafer) and matrix background spectrum. The intensity of Au was normalized by the intensity of $\mathrm{Ca}$ for a semiquantitative relative comparison. Calcium is ubiquitous in plant tissue,(36) and relatively evenly distributed along plant root apexes (observed by $\mu$-XRF mapping, data not shown). The relative standard deviation of each emission line intensity was calculated from the background noise measured after and before each line position ( $\Delta E$ of $\pm 75 \mathrm{eV}$ ).

\subsection{Hyperspectral Mapping of Au-NPs Associated with Plant Tissue (DF-HSI)}

The NPs were visualized in fresh roots using a DF-HSI system (CytoViva Inc., U.S.A.). This enhanced resolution dark-field microscope system (BX51, Olympus, U.S.A.) was equipped with a $150 \mathrm{~W}$ halogen light source (Fiber-Lite, Dolan-Jenner, U.S.A.), and a hyperspectral camera (CytoViva Hyperspectral Imaging System 1.4). The roots were observed at 60x and 100x magnification (immersion oil, numerical aperture 1.25 and 1.30, respectively). Hyperspectral images were acquired using $75 \%$ light source intensity and $0.25 \mathrm{~s}$ acquisition time per line. Each pixel of the hyperspectral image (datacube) contains its light reflectance spectrum ranging from 400 to $1000 \mathrm{~nm}$ with a step of $1.5 \mathrm{~nm}$.

Spectral libraries were built from datacubes of Au-NPs embedded in the gel (Figure S3) using ENVI 5.2 software (Exelis Visual Information Solutions, CO, United States), as described in an earlier article(17) and in the SI section "Hyperspectral Library Building". The specificity of the Au-NP libraries was confirmed by mapping Au-NPS in negative controls (gels without Au-NPs, control roots), resulting in no matches (SI Figure S3).

The Au-NP libraries were used to map the Au-NPs in hyperspectral images of dosed roots. A spectral angular mapping algorithm (SAM, ENVI 5.2) was used to identify the pixels in the dosed roots matching the Au-NP library spectra (angles $\leq 0.085$ rad were considered similar). Each pixel in the images matching the hyperspectral signature of Au-NPs was highlighted in red. All the hyperspectral images were acquired at cross-section focus. Because of the narrow 
depth of field (less than a $\mu \mathrm{m}$ ), signals of Au-NPs were only mapped by SAM in the focus plane inside of cells, and out-of-focus Au-NPs adsorbed on top or under the cells were not mapped, in agreement with previous studies. $(18,24,25)$

\subsection{D X-ray Imaging of Control and Exposed Roots by Nano-CT: Acquisition, Histogram Normalization, and Image Analysis}

Gold beads (1.2 $\pm 0.3 \mu \mathrm{m}$ in diameter, $99.96 \%$ in purity, Alfa Aesar, A. Johnson Matthey Company, U.S.A.) were deposited on the mounted root using a prealignment microscope (Zeiss, Pleasanton, U.S.A.). The gold beads served for post-treatment of sample drift correction. Nano-CT of the roots (control root and roots exposed to $10 \mathrm{mg} \cdot \mathrm{L}^{-1}$ of (干) Au-NPs) was performed using an UltraXRM-L200 X-ray microscope (Zeiss, U.S.A.) equipped with a rotating anode $\mathrm{X}$-ray source $(\mathrm{Cu}, \mathrm{K \alpha}, 8048 \mathrm{keV}$, acceleration voltage of $40 \mathrm{kV}$, current $30 \mathrm{~mA})$ and Fresnel zone plates allowing imaging at $150 \mathrm{~nm}$ spatial resolution. 901 projections were acquired in absorption contrast imaging mode, during a $180^{\circ}$ rotation of the sample (angle step of $0.19^{\circ}$ ), for a total scan time of $17 \mathrm{~h}$ (exposure time of 60s/projection). The field of view of each scan was of $65 \times 65 \times 65 \mu \mathrm{m}^{3}$ with an isotropic voxel (i.e., 3D pixel) of $63.5 \mathrm{~nm}$ $(x=y=z)$. The resulting 901 projected images of each scan were corrected for vertical and horizontal drifts of the sample during acquisition by tracking the gold bead position using the software Autoalign 2.0.4 (Xradia Inc., U.S.A.). The volume reconstruction of the $901 \mathrm{drift}$ corrected projections was performed using the nano-CT built-in software XMReconstructedParallel beam-9.0.6445 (Xradia Inc., U.S.A.) using a filtered back-projection algorithm. The software package Avizo Fire 8 (FEl, U.S.A.) was used for visualization of reconstructed data (volume rendering, virtual 2D slice), gray level histogram extraction and normalization, segmentation, and any further image analysis.

Histograms showing the number of voxels at each different gray level value were extracted from each reconstructed image. Each gray level ranging from 0 to 65535 can be attributed to an X-ray attenuation intensity that is proportional to the electronic density of the scanned materials in the sample. Here, three to four different materials were expected from X-ray absorption contrast: air, root tissue, gold, and Au-NPs. The contributions in the total histogram of (i) air, (ii) root tissue (also containing some air), and (iii) gold beads were identified by selecting subvolumes of each material in the 3D images, and extracting the corresponding histograms (see example in Figure S5).

The gray levels in the histograms are relative values that vary between different measurements. To normalize these histograms for comparisons, histograms of the air surrounding the root volume were used as an internal standard to normalize the gray levels 
between different measurements. Details on this normalization procedure are provided in the SI section "Histogram Gray Level Normalization".

The voxels containing Au-NPs were isolated based on a segmentation procedure: The gray level threshold above which the X-ray attenuation was due to Au-NPs was set to the maximum gray level value observed in the control root histogram (Figure 1, black dotted line). All voxels in Au-NP exposed roots volumes above this threshold were operationally defined to contain Au-NPs.

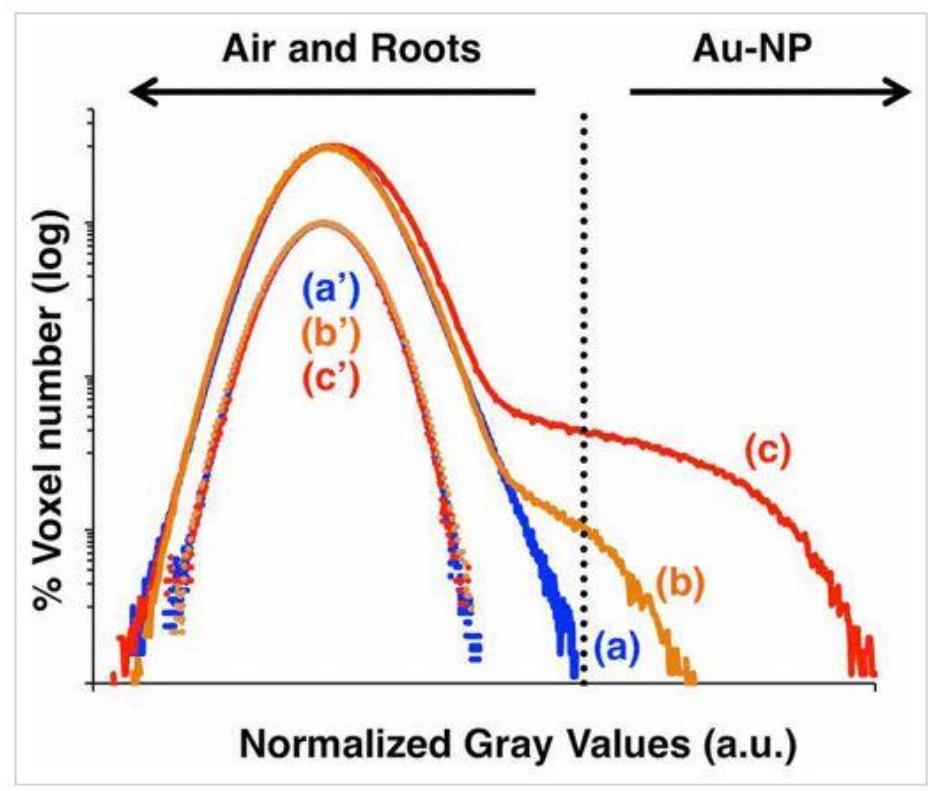

Figure 1. Normalized histograms of the root tissues subvolumes of the control (a in blue), or (F)Au-NP exposed roots ( $b$ in yellow and $c$ in red, respectively), and their respective extracted air subvolumes $\left(a^{\prime}, b^{\prime}, c^{\prime}\right)$. The Gaussians of the air subvolumes have equal means $\mu_{\text {air, control }}=\mu_{\text {air, },(-) A u-N P s}=\mu_{\text {air, },+() A u-N P s .}$ Dotted line: gray level threshold used to identify Au-NPs in the normalized histograms.

\section{Results}

\subsection{Growth and Elemental Microanalysis of Roots}

After 10 days of growth, the germination rate of $A$. thaliana was 8 out of 10 for the control plants, and 6 out of 10 and 7 out of 10 for plants exposed to (-) or (+)Au-NP, respectively (not significantly different, ANOVA, $p>0.05)$. After 10 days, the average root length and their standard deviation were $2.47 \pm 0.47 \mathrm{~cm}$ for the control root, $2.60 \pm 0.57 \mathrm{~cm}$ for the (-)Au-NP exposed root and $4.25 \pm 2.37 \mathrm{~cm}$ for the (+)Au-NP exposed root. 
$\mu$-X-ray fluorescence (spot analysis of $400 \mu \mathrm{m}$ ) was primarily used to detect the presence of $\mathrm{Au}$ associated with the roots. Two zones were analyzed: the apical zone including the root cap, and the root tip $\sim 1 \mathrm{~cm}$ above the elongation zone. No Au was detected in the control roots. In both ( $\mp$ )Au-NP exposed roots, Au was detected (Table 1 and Figure S2). When normalizing the Au XRF signal by the Ca signal, the Au distribution within the root was different for the two Au-NPs. While the Au signal of the (-)Au-NP exposed root was equally distributed between the two analyzed regions, Au in the (+)Au-NPs exposed root was mostly detected in the apical zone, while in the elongation zone, the Au signal was 5 times lower.

Table 1. Normalized $\mu$-XRF Intensities (XRF, a.u.) in Roots of Arabidopsis thaliana, Means and Standard Deviations ${ }^{a}$

\begin{tabular}{|lll|}
\hline sample & XRF emission line; & normalized intensity (a.u.) \\
treatment & zone analyzed & Au La at $1.9713 \mathrm{eV}$ \\
control root apical & n.d. \\
& elongation & n. d. \\
(-)Au-NP & apical & $1.9 \pm 0.2(\mathrm{ab})$ \\
& elongation & $1.6 \pm 0.1$ (b) \\
(+)Au-NP & apical & $2.4 \pm 0.2(\mathrm{a})$ \\
& elongation & $0.5 \pm 0.1$ (c) \\
\hline
\end{tabular}

a The roots were analyzed in (i) the apical zone including the root cap; and (ii) $\sim 1 \mathrm{~cm}$ above the root tip in the elongation zone. n.d.: not detected. The limit of detection was 0.2 a.u. Groups presenting different letters $(a, b, c)$ are significantly different (ANOVA, Turkey HSD test, $p<0.05)$.

\subsection{Hyperspectral Detection of Au-NPs in Gel and in Exposed Roots}

The collected spectral libraries of the $(\mp)$ Au-NPs were clearly distinguishable from libraries obtained from the gel culture medium with no NPs (Figures 2 and S3). The specificity of the $( \pm$ )Au-NPs libraries was assessed by mapping spectral libraries on negative controls (Figure S3). The spectral libraries of ( $\mp$ )Au-NPs differed in terms of mean absorption maxima ((-)AuNPs: $633.8 \pm 8.0 \mathrm{~nm}$; (+)Au-NPs: $605.2 \pm 1.8 \mathrm{~nm}$ ), and reflectance intensities, being $\sim 2$ times lower for (-)Au-NP library despite the similar Au-NP concentrations in gel. 


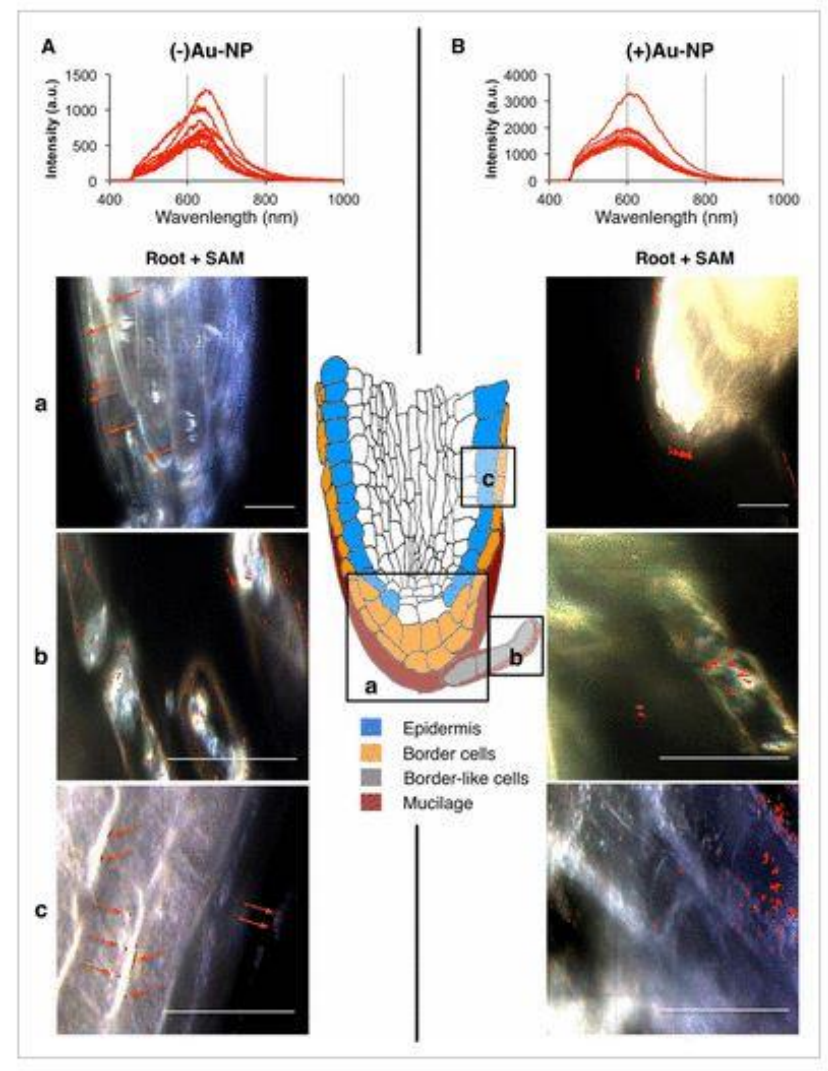

Figure 2. Spectral libraries used for the nanomaterial mapping of A. (-)Au-NPs and B. (+)AuNPs. a-c: Dark-field microscopy images of Arabidopsis thaliana roots exposed to $10 \mathrm{mg} \cdot \mathrm{L}^{-1}$ of (-)Au-NPs (left), and (+)Au-NPs (right). Red pixels: (Ғ)Au-NPs mapped using the spectral angular mapping algorithm (SAM; $0.085 \mathrm{rad}$ ). Images of different root compartments as illustrated in the top root scheme are shown. a: root cap with border-like cells and mucilage. b: detaching border-like cells. c: lateral root cap and epidermis. More hyperspectral images and SAM results of control or exposed cells are shown in Figures S3 and S4. At least 10 datacubes were acquired in 2 plant replicates per treatment. All images were acquired in cross-section focus. Scale bar: $20 \mu \mathrm{m}$.

For all roots (control and exposed), DF-HSI allowed visualizing the root architecture.(37) Note the yellow transparent layer outside of root cap cells, especially in Figure $2 a+b$ in $(+) A u-N P$ exposed roots. This layer is known as border cell mucilage consisting of negatively charged secretions that form a root extracellular trap.(38) In all cases, some detaching border-like cells (i.e., sheets of border cells, still attached but detaching from the root tip) were visualized (see Figures 2, S3, and S4).

The coating of the (干)Au-NPs visibly affected their distribution along the roots. The (+)Au-NPs mainly accumulated in the symplast and on the detaching border-like cells, outside the root mucilage (transparent brown layer visible around the root cap), and on the symplast and in 
the epidermal cells (Figure $2 a+, b+$, and $c+$ ). A thick root mucilage layer was visible in the (+)AuNP exposed root (Figure 2a+), and mucilage was abundant around their border-like cells (Figure $2 b+$ ). For the (-)Au-NP exposed root, some Au-NP were also associated with the detaching border-like cells. No root cap mucilage layer was visible (Figure 2c-), and no NPs adsorbed to it. Overall, less (-)Au-NPs than (+)Au-NPs was detected in all the root compartments. Unlike (+)Au-NPs, (-)Au-NPs were rarely localized in cells symplast, and instead mostly lined up along cell walls or lateral cell junctions and along the walls of borderlike cells. Further, the (+)Au-NPs were preferably found in larger accumulations (either agglomerates or aggregates) than the (-)Au-NPs. Similar observations were made in two different roots for each treatment (see Figure $\$ 4$ for replicates).

\subsection{D-location of Au-NPs Accumulation Zones in Roots by nano-CT}

The (+)Au-NP exposed root histogram (Figure 1C, (c)) exhibited a significantly larger fraction of denser voxels attributable to (+)Au-NPs than the (-)Au-NP exposed root histogram. The volume renderings and virtual 2D sections (cross and lateral sections) of the reconstructed nano-CT images of control and ( $\mp$ )Au-NP exposed $A$. thaliana root tips (the first $\sim 60 \mu \mathrm{m}$ of the root) are shown in Figure 3. The voxels isolated by thresholding and attributed to Au-NPs are highlighted in red (constant color). The images show the root cap, the dried mucilage on the lateral and root cap cells, and the first cells of the apical meristem above the root cap (for more explanation on the plant anatomy refer to the Figure S2).

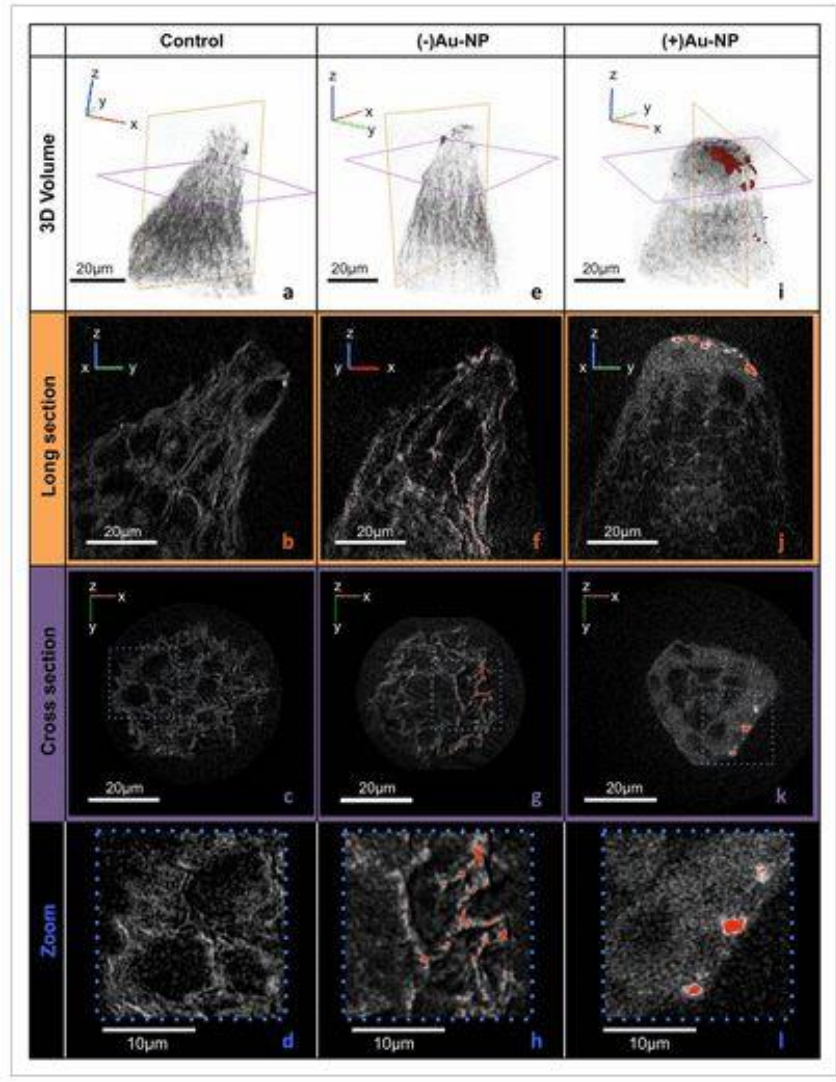


Figure 3. Reconstructed nano-CT images of $A$. thaliana root apexes. Pixels and voxels attributable to Au-NPs are shown in red. a-d: control. e-h: (-)Au-NP exposed. i-l: (+)Au-NP exposed. a, e, i: Volume renderings. b, f, j (orange): Virtual long section. c, g, k (purple): Virtual cross section $\sim 10 \mu \mathrm{m}$ above the root tip. $d, h$, l: zoom. Scale bar $=20 \mu \mathrm{m}$, voxel/pixel size $=$ $63.5 \mathrm{~nm}$. The above shown virtual cross sections are averages of 20 slices.

The volume renderings of the control, and roots exposed to (-) or (+)Au-NPs (Figure 3a,e, (i) indicate slightly different morphology. The control root and the (-)Au-NPs exposed root exhibited little or no mucilage associated with border-like cells retracting around the root tip. The two type of NPs were located differently within the roots: the (+)Au-NPs mainly remained trapped as large agglomerates in the outermost root mucilage layer, which had dried during the supercritical point drying to a relatively electron dense uniform coating around the outermost root cap cells (Figure $3 \mathrm{i}-\mathrm{k}$ ). In agreement with the DF-HSI results, this mucilage layer was remarkably thicker and slightly denser in roots exposed to (+)Au-NPs compared with the other roots, as apparent from the round, cap-like amorphous layer. No (+)Au-NPs were observed inside the root. At the opposite, the (-)Au-NP were almost exclusively distributed inside the root as small accumulation zones between cells, near cell walls or intracellular spaces (Figure $3 \mathrm{f}-\mathrm{h}$ ), all along the scanned volume.

\section{Discussion}

The present work provides direct evidence that detached border-like cells and plant mucilage can trap and accumulate large amounts of both negatively or positively charged NPs. Contrariwise, border cells on the root cap behaved charge-specific: They largely adsorbed positively charged NPs preventing further uptake or translocation, but allowed passage of negatively charged NPs into the apoplast. These new mechanistic insights on differential interactions of charged Au-NPs with roots confirm previous hypotheses about the uptake mechanisms of NPs in plants at the cellular level. Our study also demonstrates that the coupling of the two emerging techniques nano-CT and DF-HSI extends our capability to detect NPs in complex media, here plant tissues. With minimal sample preparation, we were able to screen large volumes of samples for NPs mapping in the plant tissue, in 3D using X-ray attenuation, or in 2D using light scattering information.

\subsection{Au-NPs Border-Like Cell Mucilage Trapping and Immobilization Mechanism}

In the combined results of DF-HSI and nano-CT, we observed a charge-dependent trapping process of NPs, which was probably mediated by border-like cells and mucilage. This is 
supported by the facts that (i) both ( $\mp$ )Au-NP were strongly associated with border-like cells (Figure 2b), (ii) (+)Au-NP were mainly trapped in the associated thick root tip mucilage, (Figures $2 \mathrm{a}$ and $3 \mathrm{i}-\mathrm{I}$ ), and (iii) a higher (+)Au-NP concentration was in the apical zone than in the elongation zone (Table 1). It is known that upon contact with water, plant roots release border cells as individual cells(38) or sheets of attached cells, called border-like cells in $A$. thaliana.(37) These cells remain alive several days after being separated from the root and secrete mucilage, acting as an interface between roots and the surrounding environment.(38) Root cap and border cells can trap plant pathogens,(38) sense microbes and are involved in plant immune response.(39) Mucilage-producing border cells can also trap metals such as $\mathrm{Cu}, \mathrm{Ni}, \mathrm{Zn}, \mathrm{Fe}, \mathrm{Cd}$, and $\mathrm{Al},(40-44)$ and were observed to interact with silver NPs in alfalfa (Medicago sativa)(45) or $A$. thaliana(46) and CuO-NPs in wheat (Triticum aestivum).(47)

Both imaging techniques show that both $(\mp)$ Au-NPs were stuck in the mucilage of detaching border-like cells and internalized in the root cap. Clearly, negative charges on the NP surface did not prevent interactions with detached root border-like cells, whose surface and mucilage should be mostly negatively charged at the exposure $\mathrm{pH} .(48,49)$ Furthermore, the washing procedures prior to analysis (in the case of nano- $\mathrm{CT}$ : $\mathrm{KCl}$ rinsing, PPB rinsing, glutaraldehyde fixation, several ethanol bathes, and supercritical point drying) did not remove all the NPs present at the root surface, demonstrating a strong NP sorption to the root cell surface. Electrostatic repulsions, which are often proposed in the literature to govern NP adsorption on roots, $(4,6)$ were apparently overridden in (-)Au-NP trapping by root-like border cells. Other physicochemical interactions probably promote the NP-root sorption/uptake process as well, such as diffusion into micropores of the mucilage polymers, or hydrogen or covalent bonds.(2) Further investigation on the effects of eventual $\mathrm{pH}$ variations, mucilage composition change, and anion release due to stress(49) on NP trapping is necessary to better understand and predict NP-mucilage interaction.

We also hypothesized a pronounced biological response of the roots in the presence of $(+) A u-$ NPs, compared to the controls or to the roots exposed to (-)Au-NPs. Indeed, we observed in three roots per treatment, both in DF-HSI (on two replicates) and nano-CT, an abundant and thick mucilage on the (+)Au-NP exposed root cap that resisted to root washing and fixation in the preparation of samples. Even if all roots were treated in the same way, this mucilage was less pronounced on the root cap of controls or (-)Au-NPs exposed roots. The amount and composition of root mucilage is known to vary as response to some stresses.(49) Aluminum for example induces a thicker mucilage layer around detached border cells of snapbeans, Phaseolus vulgaris.(41) As response to the pathogen Nectria hematococca, the 
root cap of pea secretes a complex mixture of proteins in addition to the usual range of high molecular weight polysaccharides.(50) In our case, besides other possible defense strategies,(51) $A$. thaliana roots may have altered its mucilage production or composition as a response to (+)Au-NP exposure to protect(38) the sensitive root tip.

Finally, the DF-HSI imaging shows that border cells can play an important role in NP-plant interactions by adsorbing NPs. These observations could at least partially explain contradictory results observed in previous studies dealing with NP-roots interactions in hydroponics.(52) Under hydroponic conditions, border cells often detach from the roots.(53) The lack of border cells, and thus of mucilage, may fundamentally change the NP uptake and the response of plant to NPs. In the present work, the plant growth medium was a gel(54) that allowed for normal development and production of border-like cells,(37) providing more realistic insight on NP-root interaction than hydroponic systems. Furthermore, in a real rhizosphere, the presence of additional phases (e.g., humic acids, microorganisms, and so forth) could alter NP-coating or induce NP transformations, $(23,55$, 56) ultimately influencing mucilage production and NP-root interactions. These effects have not been assessed here. Nevertheless, the present results show that gel used as a plant growth medium can provide realistic insights into NP-root interaction mechanisms for terrestrial plants in solid matrices.

\subsection{Direct Evidence for Charge-Specific Au-NP Translocation Mechanisms}

Previous studies assessing the charge effect of Au-NP on root interaction already showed a strong colocation of Au-NPs and root tips, without being able to determine if Au-NP were adsorbed on or accumulated in the root. $(4,5)$ In the present study, charge-specific NP uptake into the apoplast was observed both with DF-HSI and nano-CT (Figure 2, Figure 3). The (+)AuNPs were rarely observed in the cells (symplast, Figure 2). On the contrary, (-)Au-NPs were detected in the apoplast, i.e., within the cell walls and extracellular spaces. This supports previous literature hypotheses on the effects of NP charge on their uptake and transport in plants, where transport of negatively charged NPs was facilitated-much like for large metal complexes(57)-along the apoplast.(2) Positively charged NP uptake seems to happen to a small extent, possibly by other endocytic processes such as clathrin receptor-mediated endocytosis. $(2,58,59)$

In addition to surface charge, agglomeration may affect NP translocation. The apparent good dispersion of the (-)Au-NPs in the gel substrate of the plant may have favored uptake. The agglomeration of the (+)Au-NPs in the (negatively charged) gel (Figure S3), even in absence of plant exudates, may have limited their mobility and uptake. Theoretically, the different hydrodynamic diameter as well as the nature of the coating of the two (₹)Au-NPs (18.6 and 
$47.6 \mathrm{~nm}$, respectively) may have affected their uptake behavior as well. The hydrodynamic diameter of (-)Au-NPs was too large to allow any passage through NP-permeable plasmodesmata microchannels in the symplast $(<3-4 \mathrm{~nm})$, but small enough to pass NPpermeable longitudinal channels in cell walls in the apoplast in the vasculature of $<36-50$ $\mathrm{nm} .(2,7)$ In contrast, based on their hydrodynamic diameter, the (+)Au-NPs were too big to cross both symplast and apoplast channels. Thus, the hydrodynamic diameter could have favored uptake and translocation of (-)Au-NPs. However, abundant (+)Au-NPs were present in the cytoplasm of border-like cells Figure $2 c$ (acquired in cross-section focus), showing that the NPs were able to cross cell walls despite their larger hydrodynamic diameter. The hydrodynamic diameter-including flexible surface coating polymers and the water hydration shell-seemed of less importance here than the TEM diameter of the solid Au core.

It is not entirely clear if the root cells induced the observed NP agglomeration actively due to stress, or if a passive physicochemical sorption process occurred. Previous studies showed that positively charged Au-NPs were associated with a greater extent with plant roots than negatively charged Au-NPs, which then translocate to a greater extent into the above-ground tissue.(60) The present results indicate that the underlying mechanisms can include active or passive extracellular trapping of NPs.

\subsection{Complementary Coupling of DF-HSI and Nano-CT}

The distribution of $(\bar{\mp})$ Au NPs in roots was assessed using two emerging tools, DF-HSI, and nano-CT. The results obtained with the two techniques (in total three plants per treatment) are consistent, which supports the validity and robustness of the observations.

The main advantage of DF-HSI is that little sample preparation is required (the rinsed roots were directly mounted on a glass slide), avoiding artifacts due to typical sample preparation steps required for most imaging techniques (e.g., labeling, staining, drying, icing, or cutting steps). As an example, we were able to detect Au NPs on detached border cells, which would probably have been lost during any more destructive sample preparation. Another advantage is the specificity of the reflectance signal from the NPs. A drawback is that the obtained pictures capture the light scattering of the material, and a statistical analysis of the size of the small objects is impossible. Furthermore, the obtained images were 2-dimensional, which, in some cases, can confound potential internalization.

Nano-CT has previously been described as promising for the imaging of living tissue architecture and morphology.(61) However, its use in laboratory is still scare, and, to our knowledge, this study is the first one assessing its use for NP detection in unstained tissues. This tool benefits from the high penetrating properties of $\mathrm{X}$-rays through matter that allows 
the $3 \mathrm{D}$ reconstruction of a volume from a stack of $2 \mathrm{D} X$-ray images. This approach may also be used for denser matrices.(62) Similar to DF-HSI, which does not provide information about the size of the objects, nano-CT voxel size was bigger than the NPs unit size. Therefore, in this specific scenario, we cannot draw conclusions about the aggregation state of the NPs. However, in contrast to DF-HSI, the entire analyzed 3D volume of the root can be sliced into a multitude of 2D sections, providing important information about NP internalization or adsorption into/onto the tissues at the micro- and nanoscale. It is important to note that the sample needs to be dried prior to analysis to avoid drying effects under the beam that would alter the quality of the 3D image. The drying could theoretically dislocate NP to other compartments (e.g., symplast vs apoplast). However, the results obtained with the two techniques are consistent, which suggests that the supercritical point drying does not drastically affect the root architecture and the Au-NP distribution.

The use of two complementary tools improved our comprehension of AuNP-roots interactions. Limitations for DF-HSI mainly exist for opaque matrices because of more scattering of the visible light compared to X-rays. For instance, we recently used micro-CT for the characterization of photocatalytic cement pastes,(63) whose internal structure cannot be probed by DF-HSI. Limitations for X-ray CT exist for NPs possessing low electron density, and thus its detection limit mostly depends on the X-ray absorption contrast between the NPs and the matrix. Some questions also remain on the detection and quantification limits of these tools, which could be affected by NP transformations such as dissolution, oxidation, phase transformation, ion release, and agglomeration/aggregation. Although the sensitivity of both nano-CT and DF-HSI is system-dependent and should be further investigated in more complex systems and using chemically less inert NPs, this approach will be applicable to other systems, including other matrices and nanoparticle types.

The authors declare no competing financial interest.

\section{Acknowledgment}

This work is a contribution to the Labex Serenade (no. ANR-11-LABX-0064) funded by the "Investissements d'Avenir" French Government program of the French National Research Agency (ANR) through the A*MIDEX project (no ANR-11-IDEX-0001-02) and the French X-ray CT platform called Nano-ID was funded by the EQUIPEX project ANR-10-EQPX-39-01. F.S. was supported by the Swiss NSF (P300P3-158517). 


\section{References}

1.Gardea-Torresdey, J. L.; Rico, C. M.; White, J. C. Trophic Transfer, Transformation, and Impact of Engineered Nanomaterials in Terrestrial Environments

Environ. Sci. Technol. 2014, 48 (5) 2526- 2540 DOI: 10.1021/es4050665

2.Schwab, F.; Zhai, G.; Kern, M.; Turner, A.; Schnoor, J. $\quad$ L.; Wiesner, M. R. Barriers, pathways and processes for uptake, translocation and accumulation of nanomaterials in plants - Critical review

Nanotoxicology 2016,10 (3) 257- 278 DOI: 10.3109/17435390.2015.1048326

3.Tripathi, D. $\quad$ K.; Shweta; Singh, S.; Singh, S.; Pandey, R.; Singh, V. $\quad$ P.; Sharma, N. C.; Prasad, S. M.;Dubey, N. K.; Chauhan, D. K. An overview on manufactured nanoparticles in plants: Uptake, translocation, accumulation and phytotoxicity

Plant Physiol. Biochem. 2017, 110, 2 DOI: 10.1016/j.plaphy.2016.07.030

4.Judy, J. D.; Unrine, J. M.; Rao, W.; Wirick, S.; Bertsch, P. M. Bioavailability of Gold Nanomaterials to Plants: Importance of Particle Size and Surface Coating

Environ. Sci. Technol. 2012, 46 (15) 8467-8474 DOI: 10.1021/es3019397

5.Koelmel, J.; Leland, T.; Wang, H.; Amarasiriwardena, D.; Xing, B. Investigation of gold nanoparticles uptake and their tissue level distribution in rice plants by laser ablationinductively coupled-mass spectrometry

Environ. Pollut. 2013, 174, 222- 228 DOI: 10.1016/j.envpol.2012.11.026

6.Zhu, Z.J.; Wang, H.; Yan, B.; Zheng, H.; Jiang, Y.; Miranda, O.R.; Rotello, V.M.; Xing, B.; Vachet, R. W.Effect of Surface Charge on the Uptake and Distribution of Gold Nanoparticles in Four Plant Species

Environ. Sci. Technol. 2012, 46 (22) 12391- 12398 DOI: 10.1021/es301977w

7.Larue, C.; Laurette, J.; HerlinBoime, N.; Khodja, H.; Fayard, B.; Flank, A.M.; Brisset, F.; Carriere, M.Accumulation, translocation and impact of $\mathrm{TiO} 2$ nanoparticles in wheat (Triticum aestivum spp.): influence of diameter and crystal phase

Sci. Total Environ. 2012, 431, 197- 208 DOI: 10.1016/j.scitotenv.2012.04.073

8.Ma, X.; Quah, B. Effects of Surface Charge on the Fate and Phytotoxicity of Gold Nanoparticles to Phaseolus vulgaris Food Chem. Nanotechnol 2016, 2 (1) 57-65 DOI: 10.17756/jfcn.2016-011

9.Reddy, P. V. L; Hernandez-Viezcas, J. A.; Peralta-Videa, J. R.; Gardea-Torresdey, J. L. Lessons learned: Are engineered nanomaterials toxic to terrestrial plants?

Sci. Total Environ. 2016, 568 (568) 470- 479 DOI: 10.1016/j.scitotenv.2016.06.042

10.Zhang, P.; Ma, Y.; Zhang, Z. Interactions Between Engineered Nanomaterials and Plants: Phytotoxicity, Uptake, Translocation, and Biotransformation. In Nanotechnology 
and Plant Sciences; Siddiqui, M. H.;Al-Whaibi, M. H.; Mohammad, F., Eds.; Springer International Publishing: New York, 2015; pp 77-99.

11.Bao, D.; Oh, Z. G.; Chen, Z. Characterization of Silver Nanoparticles Internalized by Arabidopsis Plants Using Single Particle ICP-MS Analysis

Front. Plant Sci. 2016, 7, 32 DOI: 10.3389/fpls.2016.00032

12..Petersen, EJ.; Henry, TB.; Zhao, J.; MacCuspie, R.I.; Kirschling, TL.; Dobrovolskaia, M. A.; Hackley, V.;Xing, B.; White, J. C. Identification and Avoidance of Potential Artifacts and Misinterpretations in Nanomaterial Ecotoxicity Measurements

Environ. Sci. Technol. 2014, 48 (8) 4226- 4246 DOI: 10.1021/es4052999

13.Ayache, J.; Beaunier, L.; Boumendil, J.; Ehret, G.; Laub, D. Artifacts in Transmission Electron Microscopy. In Sample Preparation Handbook for Transmission Electron Microscopy; Springer: New York, 2010; pp 125-170.

14.Tiede, K.; Boxall, A. B. A.; Tear, S. P.; Lewis, J.; David, H.; Hassellöv, M. Detection and characterization of engineered nanoparticles in food and the environment

Food Addit. Contam. Part A 2008, 25 (7) 795-821DOI: 10.1080/02652030802007553

15.Schultz, C.; Powell, K.; Crossley, A.; Jurkschat, K.; Kille, P.; Morgan, A.J.; Read, D.; Tyn e, W.; Lahive, E.;Svendsen, C.Analytical approaches to support current understanding of exposure, uptake and distributions of engineered nanoparticles by aquatic and terrestrial organisms

Ecotoxicology 2015, 24 (2)239- 261 DOI: 10.1007/s10646-014-1387-3

16.Vainrub, A.; Pustovyy, O.; Vodyanoy, V. Resolution of $90 \mathrm{~nm}(\lambda / 5)$ in an optical transmission microscope with an annular condenser

Opt. Lett. 2006, 31 (19) 2855 DOI: 10.1364/OL.31.002855

17. Badireddy, A. R.; Wiesner, M. R.; Liu, J. Detection, Characterization, and Abundance of Engineered Nanoparticles in Complex Waters by Hyperspectral Imagery with Enhanced Darkfield Microscopy

Environ. Sci. Technol. 2012, 46 (18) 10081- 10088 DOI: 10.1021/es204140s

18.Vetten, M.A.; Tlotleng, N.; TannerRascher, D.; Skepu, A.; Keter, F.K.; Boodhia, K.; Koe kemoer, L.-A.;Andraos, C.; Tshikhudo, R.; Gulumian, M. Label-free in vitro toxicity and uptake assessment of citrate stabilised gold nanoparticles in three cell line

Part. Fibre Toxicol. 2013, 10, 50 DOI: 10.1186/1743-8977-10-50

19.Roth, G.A.; SosaPeña, M.; del, P.; Neu-Baker, N.M.; Tahiliani, S.; Brenner, SA.

Identification of Metal Oxide Nanoparticles in Histological Samples by Enhanced Darkfield Microscopy and Hyperspectral Mapping

J. Visualized Exp. 2015, 106, 53317 DOI: 10.3791/53317 
20.Mortimer, M.; Gogos, A.; Bartolomé, N.; Kahru, A.; Bucheli, T.D.; Slaveykova, V.I. Pot ential of Hyperspectral Imaging Microscopy for Semi-quantitative Analysis of Nanoparticle Uptake by Protozoa

Environ. Sci. Technol. 2014, 48 (15) 8760- 8767 DOI: 10.1021/es500898j

21.Badireddy, A. R.; Farner Budarz, J.; Marinakos, S. M.; Chellam, S.; Wiesner, M. R. Formation of Silver Nanoparticles in Visible Light-Illuminated Waters: Mechanism and Possible Impacts on the Persistence of AgNPs and Bacterial Lysis

Environ. Eng. Sci. 2014, 31 (7) 338- 349 DOI: 10.1089/ees.2013.0366

22.Avellan, A.; Levard, C.; Rose, J.; Auffan, M.; Bertrand, M.; Olivi, L.; Santaella, C.; Acho uak, W.; Masion, A.Influence of structural defects of Ge-imogolite nanotubes on their toxicity towards Pseudomonas brassicacearum

Environ. Sci.: Nano 2016, 3 (4) 839- 846 DOI: 10.1039/C6EN00060F

23.Avellan, A.; Auffan, M.; Masion, A.; Levard, C.; Bertrand, M.; Rose, J.; Santaella, C.; A chouak, W. Remote Biodegradation of Ge-Imogolite Nanotubes Controlled by the Iron Homeostasis of Pseudomonas brassicacearum

Environ. Sci. Technol. 2016, 50 (14) 7791- 7798 DOI: 10.1021/acs.est.6b01455

24.Schwab, F.; Bucheli, T.D.; Camenzuli, L.; Magrez, A.; Knauer, K.; Sigg, L.; Nowack, B. D iuron Sorbed to Carbon Nanotubes Exhibits Enhanced Toxicity to Chlorella vulgaris

Environ. Sci. Technol. 2013, 47 (13)7012- 7019 DOI: 10.1021/es304016u

25.Leclerc, S.; Wilkinson, K. J. Bioaccumulation of Nanosilver by Chlamydomonas reinhardtii-Nanoparticle or the Free Ion?

Environ. Sci. Technol. 2014, 48 (1) 358- 364 DOI: 10.1021/es404037z

26.Kwok, K. W. H.; Auffan, M.; Badireddy, A. R.; Nelson, C. M.; Wiesner, M. R.; Chilkoti, A.; Liu, J.;Marinakos, S. M.; Hinton, D. E. Uptake of silver nanoparticles and toxicity to early life stages of Japanese medaka (Oryzias latipes): Effect of coating materials

Aquat. Toxicol. 2012, 120-121, 59-66 DOI: 10.1016/j.aquatox.2012.04.012

27.Meyer, J. N.; Lord, C. A.; Yang, X. Y.; Turner, E. A.; Badireddy, A. R.; Marinakos, S. M.; Chilkoti, A.;Wiesner, M. R.; Auffan, M. Intracellular uptake and associated toxicity of silver nanoparticles in Caenorhabditis elegans

Aquat. Toxicol. 2010, 100 (2) 140- 150 DOI: 10.1016/j.aquatox.2010.07.016

28.Yang, X.; Jiang, C.; Hsu-Kim, H.; Badireddy, A. R.; Dykstra, M.; Wiesner, M.; Hinton, D. E.; Meyer, J. N.Silver Nanoparticle Behavior, Uptake, and Toxicity in Caenorhabditis elegans: Effects of Natural Organic Matter

Environ. Sci. Technol. 2014, 48 (6) 3486- 3495 DOI: 10.1021/es404444n

29.Dhondt, S.; Vanhaeren, H.; VanLoo, D.; Cnudde, V.; Inzé, D. Plant structure visualization by high-resolution $\mathrm{X}$-ray computed tomography 
Trends Plant Sci. 2010, 15 (8) 419-422 DOI: 10.1016/j.tplants.2010.05.002

30.Yin, G.C.; Tang, M.T.; Song, Y.F.; Chen, F.R.; Liang, K.S.; Duewer, F.W.; Yun, W.; Ko, C. -H.;Shieh, H.-P. D. Energy-tunable transmission $\mathrm{x}$-ray microscope for differential contrast imaging with near $60 \mathrm{~nm}$ resolution tomography

Appl. Phys. Lett. 2006, 88 (24) 241115 DOI: 10.1063/1.2211300

31.vonHofsten, O.; Bertilson, M.; Reinspach, J.; Holmberg, A.; Hertz, H.M.; Vogt, U. Sub25-nm laboratory $\mathrm{x}$-ray microscopy using a compound Fresnel zone plate

Opt. Lett. 2009, 34 (17) 2631- 2633 DOI: 10.1364/OL.34.002631

32.Sakdinawat, A.; Attwood, D. Nanoscale X-ray imaging

Nat. Photonics 2010, 4 (12) 840-848 DOI: 10.1038/nphoton.2010.267

33.Santaella, C.; Schue, M.; Berge, O.; Heulin, T.; Achouak, W. The exopolysaccharide of Rhizobium sp. YAS34 is not necessary for biofilm formation on Arabidopsis thaliana and Brassica napus roots but contributes to root colonization

Environ. Microbiol. 2008, 10 (8) 2150- 2163 DOI: 10.1111/j.1462-2920.2008.01650.x

34.Schneider, C. A.; Rasband, W. S.; Eliceiri, K. W. NIH Image to ImageJ: 25 years of image analysis

Nat. Methods 2012, 9 (7) 671-675 DOI: 10.1038/nmeth.2089

35. Horridge, G. A.; Tamm, S. L. Critical Point Drying for Scanning Electron Microscopic Sthdy of Ciliary Motion

Science 1969, 163 (3869) 817- 818 DOI: 10.1126/science.163.3869.817

36. White, P. J. The pathways of calcium movement to the xylem

J. Exp. Bot. 2001, 52 (358) 891-899 DOI: 10.1093/jexbot/52.358.891

37.Vicré, M.; Santaella, C.; Blanchet, S.; Gateau, A.; Driouich, A. Root Border-Like Cells of Arabidopsis. Microscopical Characterization and Role in the Interaction with Rhizobacteria

Plant Physiol. 2005, 138 (2)998-1008 DOI: 10.1104/pp.104.051813

38.Driouich, A.; Follet-Gueye, M.-L.; Vicré-Gibouin, M.; Hawes, M. Root border cells and secretions as critical elements in plant host defense

Curr. Opin. Plant Biol. 2013, 16 (4) 489- 495 DOI: 10.1016/j.pbi.2013.06.010

39.Plancot, B.; Santaella, C.; Jaber, R.; KieferMeyer, M.C.; FolletGueye, M.L.; Leprince, J. ; Gattin, I.;Souc, C.; Driouich, A.; Vicré-Gibouin, M. Deciphering the Responses of Root Border-Like Cells of Arabidopsis and Flax to Pathogen-Derived Elicitors

Plant Physiol. 2013, 163 (4) 1584- 1597 DOI: 10.1104/pp.113.222356

40.Kopittke, P.M.; Menzies, N.W.; deJonge, M.D.; McKenna, B. A.; Donner, E.; Webb, R. I.; Paterson, D. J.;Howard, D. L.; Ryan, C. G.; Glover, C. J.In Situ Distribution and Speciation of Toxic Copper, Nickel, and Zinc in Hydrated Roots of Cowpea 
Plant Physiol. 2011, 156 (2) 663- 673 DOI: 10.1104/pp.111.173716

41. Miyasaka, S. C.; Hawes, M. C. Possible role of root border cells in detection and avoidance of aluminum toxicity

Plant Physiol. 2001, 125 (4) 1978- 1987 DOI: 10.1104/pp.125.4.1978

42.Brigham, L. A.; Hawes, M. C.; Miyasaka, S. C. Avoidance of aluminium toxicity: Role of root border cells. In

PlantNutrition; Horst, W.J.; Schenk, M.K.; Bürkert, A.; Claassen, N.; Flessa, H.; Frommer , W. B.;Goldbach, H.; Olfs, H.-W.; Römheld, V.; Sattelmacher, B., , Eds.; Developments in Plant and Soil Sciences; Springer: Netherlands, 2001; pp 452- 453.

43.Zhang, Y.; Zheng, G. H.; Liu, P.; Song, J. M.; Xu, G. D.; Cai, M. Z. Morphological and physiological responses of root tip cells to $\mathrm{Fe} 2+$ toxicity in rice

Acta Physiol. Plant. 2011, 33 (3) 683- 689 DOI: 10.1007/s11738-010-0590-y

44.Balestri, M.; Ceccarini, A.; Forino, L. M.C.; Zelko, I.; Martinka, M.; Lux, A.;

Castiglione, M. R. Cadmium uptake, localization and stress-induced morphogenic response in the fern Pteris vittata Planta 2014, 239 (5)1055- 1064 DOI: 10.1007/s00425014-2036-z

45.Stegemeier, J.P.; Schwab, F.; Colman, B.P.; Webb, S.M.; Newville, M.; Lanzirotti, A.; Winkler, C.; Wiesner, M. R.; Lowry, G. V. Speciation Matters: Bioavailability of Silver and Silver Sulfide Nanoparticles to Alfalfa (Medicago sativa)

Environ. Sci. Technol. 2015, 49 (14) 8451- 8460 DOI: 10.1021/acs.est.5b01147

46.GeislerLee, J.; Wang, Q.; Yao, Y.; Zhang, W.; Geisler, M.; Li, K.; Huang, Y.; Chen, Y.; Ko Imakov, A.; Ma, X.Phytotoxicity, accumulation and transport of silver nanoparticles by Arabidopsis thaliana

Nanotoxicology2012, 7 (3) 323-337 DOI: 10.3109/17435390.2012.658094

47.Wright, M.; Adams, J.; Yang, K.; McManus, P.; Jacobson, A.; Gade, A.; McLean, J.; Brit t, D.; Anderson, A. A Root-Colonizing Pseudomonad Lessens Stress Responses in Wheat Imposed by $\mathrm{CuO}$ Nanoparticles

PLoS One 2016, 11 (10) e0164635 DOI: 10.1371/journal.pone.0164635

48.Meychik, N. R.; Yermakov, I. P. Ion exchange properties of plant root cell walls

Plant Soil 2001, 234 (2) 181-193 DOI: 10.1023/A:1017936318435

49.Javed, M. T.; Stoltz, E.; Lindberg, S.; Greger, M. Changes in $\mathrm{pH}$ and organic acids in mucilage of Eriophorum angustifolium roots after exposure to elevated concentrations of toxic elements

Environ. Sci. Pollut. Res. 2013, 20 (3) 1876- 1880 DOI: 10.1007/s11356-012-1413-z

50.Wen, F.; VanEtten, H. D.; Tsaprailis, G.; Hawes, M. C. Extracellular Proteins in Pea Root Tip and Border Cell Exudates 
Plant Physiol. 2006, 143 (2) 773- 783 DOI: 10.1104/pp.106.091637

51.Ma, C.; White, J. C.; Dhankher, O. P.; Xing, B. Metal-Based Nanotoxicity and Detoxification Pathways in Higher Plants

Environ. Sci. Technol. 2015, 49 (12) 7109- 7122 DOI: 10.1021/acs.est.5b00685

52.Zuverza-Mena, N.; Martínez-Fernández, D.; Du, W.; Hernandez-Viezcas, J. A.; BonillaBird, N.;López-Moreno, M. L.; Komárek, M.; Peralta-Videa, J. R.; Gardea-Torresdey, J. L. Exposure of engineered nanomaterials to plants: Insights into the physiological and biochemical responses-A review.

Plant Physiol. Biochem. 2017, 110, 236- 264. DOI: 10.1016/j.plaphy.2016.05.037

53.Pan, J.-W.; Ye, D.; Wang, L.-L.; Hua, J.; Zhao, G.-F.; Pan, W.-H.; Han, N.; Zhu, M.-

Y. Root Border Cell Development is a Temperature-Insensitive and Al-Sensitive Process in Barley

Plant Cell Physiol. 2004, 45(6) 751- 760 DOI: 10.1093/pcp/pch090

54.Arnon, D. I.; Hoagland, D. R. Crop production in artificial culture solutions and in soils with special reference to factors influencing yields and absorption of inorganic nutrients

Soil Sci. 1940, 50, 463- 485

55.Bian, S.-W.; Mudunkotuwa, I. A.; Rupasinghe, T.; Grassian, V. H. Aggregation and Dissolution of $4 \mathrm{~nm} \mathrm{ZnO}$ Nanoparticles in Aqueous Environments: Influence of $\mathrm{pH}$, Ionic Strength, Size, and Adsorption of Humic Acid

Langmuir 2011, 27 (10) 6059-6068 DOI: 10.1021/la200570n

56.Vindedahl, A. M.; Strehlau, J. H.; Arnold, W. A.; Penn, R. L. Organic matter and iron oxide nanoparticles: aggregation, interactions, and reactivity

Environ. Sci.: Nano 2016, 3 (3) 494- 505 DOI: 10.1039/C5EN00215J

57.Sarret, G.; Pilon Smits, E. A. H.; Castillo Michel, H.; Isaure, M. P.; Zhao, F. J.; Tappero, R. Use of synchrotron-based techniques to elucidate metal uptake and metabolism in plants

Adv. Agron. 2013, 119, 1-82 DOI: 10.1016/B978-0-12-407247-3.00001-9

58.von Moos, N.; Bowen, P.; Slaveykova, V. I. Bioavailability of inorganic nanoparticles to planktonic bacteria and aquatic microalgae in freshwater

Environ. Sci.: Nano 2014, 1 (3) 214 DOI: 10.1039/c3en00054k

59.Harush-Frenkel, O.; Debotton, N.; Benita, S.; Altschuler, Y. Targeting of nanoparticles to the clathrin-mediated endocytic pathway

Biochem. Biophys. Res. Commun. 2007, 353 (1) 26- 32 DOI: 10.1016/j.bbrc.2006.11.135

60.Judy, J. D.; Unrine, J. M.; Rao, W.; Bertsch, P. M. Bioaccumulation of Gold Nanomaterials by Manduca sextathrough Dietary Uptake of Surface Contaminated Plant Tissue 
Environ. Sci. Technol. 2012, 46 (22)12672- 12678 DOI: 10.1021/es303333w

61.Walton, L. $\quad$ A.; Bradley, R. S.; Withers, P. J.; Newton, V. L.; Watson, R. E. B.; Austin, C.; Sherratt, M. J.Morphological Characterisation of Unstained and Intact Tissue Micro-architecture by X-ray Computed Micro- and Nano-Tomography

Sci. Rep. 2015, 5, 10074 DOI: 10.1038/srep10074

62.Bossa, N.; Chaurand, P.; Vicente, J.; Borschneck, D.; Levard, C.; AguerreChariol, O.; Rose, J. Micro- and nano-X-ray computed-tomography: A step forward in the characterization of the pore network of a leached cement paste

Cem. Concr. Res. 2015, 67, 138- 147 DOI: 10.1016/j.cemconres.2014.08.007

63.Bossa, N.; Chaurand, P.; Levard, C.; Borschneck, D.; Miche, H.; Vicente, J.; Geantet, C .;Aguerre-Chariol, O.; Michel, F. M.; Rose, J. Environmental exposure to TiO2 nanomaterials incorporated in building material

Environ. Pollut. 2017, 220 (B) 1160- 1170 DOI: 10.1016/j.envpol.2016.11.019 